\title{
AN AFFINE INVARIANT INEQUALITY FOR RATIONAL FUNCTIONS AND APPLICATIONS IN HARMONIC ANALYSIS
}

\author{
SPYRIDON DENDRINOS, MAGALI FOLCH-GABAYET, AND JAMES WRIGHT
}

\begin{abstract}
We extend an affine invariant inequality for vector polynomials established in [6] to general rational functions. As a consequence we obtain sharp universal estimates for various problems in euclidean harmonic analysis defined with respect to the so-called affine arclength measure.
\end{abstract}

\section{IntRoduction}

In [6] the following inequality for vector polynomials was established: let $\vec{P}(t)=$ $\left(P_{1}(t), \ldots, P_{n}(t)\right)$ be an $n$-tuple of real polynomials of a single variable whose degrees are at most $d$, and set

$$
J_{\vec{P}}\left(t_{1}, \ldots, t_{n}\right)=\operatorname{det}\left(\vec{P}^{\prime}\left(t_{1}\right) \cdots \vec{P}^{\prime}\left(t_{n}\right)\right) \quad \text { and } \quad L_{\vec{P}}(t)=\operatorname{det}\left(\vec{P}^{\prime}(t) \cdots \vec{P}^{(n)}(t)\right),
$$

the determinants of $n \times n$ matrices whose $j$ th columns are given by $\vec{P}^{\prime}\left(t_{j}\right)$ and $\vec{P}^{(j)}(t)$ respectively. Then there are constants $\epsilon=\epsilon_{d, n}, M=M_{d, n}$ with $0<\epsilon, M<\infty$, so that any closed interval $I \subset \mathbb{R}$ can be decomposed $I=\cup_{\ell=1}^{N} J_{\ell}$ where the $J_{\ell}$ are closed disjoint (interiors) intervals with $N \leq M$ and for each $1 \leq \ell \leq N$,

$$
\left|J_{\vec{P}}(\vec{t})\right| \geq \epsilon \prod_{j=1}^{n}\left|L_{\vec{P}}\left(t_{j}\right)\right|^{1 / n} \prod_{1 \leq r<s \leq n}\left|t_{r}-t_{s}\right|
$$

for all $\vec{t}=\left(t_{1}, \ldots, t_{n}\right) \in J_{\ell}^{n}$. Note that (1) remains unchanged if $\vec{P}$ is replaced by any affine image $A \vec{P}+\vec{v}$ where $A \in G L_{n}(\mathbb{R})$ and $\vec{v} \in \mathbb{R}^{n}$. This affine invariance is important in applications (see [6] for an application of (1) to Fourier restriction and [7] for an application to $L^{p}$ improving bounds for averaging operators) as well as being crucial in the proof to establish (1) itself.

In this note we extend (1) to rational functions. More precisely we establish the following result.

Theorem 1.1. Let $\vec{R}(t)=\left(R_{1}(t), \ldots, R_{n}(t)\right)$ be an $n$-tuple of rational functions whose degrees do not exceed $d$ and set $J_{\vec{R}}(\vec{t})$ and $L_{\vec{R}}(t)$ as above. Then there exist positive and finite constants $\epsilon_{d, n}$ and $M_{d, n}$ so that every closed interval $I=\cup_{\ell=1}^{N} \bar{J}_{\ell}$

1991 Mathematics Subject Classification. 42B10.

The second author acknowledges financial support from CONACyT (37046-E) and DGAPAUNAM (PAPIIT IN101303).

The third author was supported in part by an EPSRC grant. 
can be decomposed into disjoint open intervals $\left\{J_{\ell}\right\}_{\ell=1}^{N}$ with $N \leq M_{d, n}$ and where (1) holds on each $J_{\ell}^{n}$.

As an application of Theorem 1.1, we have the following result in the theory of Fourier restriction to curves.

Theorem 1.2. Let $\vec{R}(t), L_{\vec{R}}(t)$ be as in Theorem 1.1 and set $w(t)=\left|L_{\vec{R}}(t)\right|^{2 / n(n+1)}$. Then for $p^{\prime}=\frac{n(n+1)}{2} q$ and $1 \leq p<\frac{n^{2}+2 n}{n^{2}+2 n-2}$,

$$
\left(\int_{\mathbb{R}}|\hat{f}(\vec{R}(t))|^{q} w(t) d t\right)^{\frac{1}{q}} \leq C\|f\|_{L^{p}\left(\mathbb{R}^{n}\right)}
$$

holds for all $f \in C_{c}^{\infty}\left(\mathbb{R}^{n}\right)$ and some $C=C_{p, d, n}$.

Remarks 1.3.

- The example $\vec{R}(t)=\left(t, t^{2}, \ldots, t^{n}\right)$ shows that the condition $p^{\prime}=n(n+$ 1) $/ 2 q$ is sharp but from the work of Drury [8] one expects the range of $p$ could be enlarged to $1 \leq p<\left(n^{2}+n+2\right) /\left(n^{2}+n\right)$.

- The measure $\omega$ on the curve parameterised by $\vec{R}(t)$ (defined on a test function $\phi$ by $\left.\omega(\phi)=\int_{\mathbb{R}} \phi(\vec{R}(t)) w(t) d t\right)$ is called the affine arclength measure and mitigates any curvature degeneracies which $\vec{R}$ may possess. One then expects (2) to hold for large classes of curves in $\mathbb{R}^{n}$ with a corresponding uniform bound $C$; this has been investigated by a number of authors, see for example, [1], [2], [6], [9], [10], [11], [13] and [15]. However simple examples show that $(2)$ can fail if $L_{\vec{R}}(t)$ changes sign too often and so the class of rational curves is natural to consider as the number of sign changes of $L_{\vec{R}}$ is controlled by $d$. Finally a nice feature is that on the critical line $p^{\prime}=n(n+1) / 2 q$, the estimate $(2)$ is affine-invariant.

- The proof of Theorem 1.2 follows the argument of M. Christ in [4], using Theorem 1.1; see [6] for details.

Another application of Theorem 1.1 lies in the area of universal $L^{p}$ improving bounds for averaging operators along curves defined with respect to the affine arclength measure $\omega$. The following theorem extends results in [14] and [7].

Theorem 1.4. With $\vec{R}$ and $\omega$ defined as above, set $\mathcal{A} f(x)=f * \omega(x)$ and $n=2$ or 3 . Then for every $\epsilon>0$,

$$
\|\mathcal{A} f\|_{L^{\frac{n^{2}+n}{2 n-2}, \frac{n+1}{2}+\epsilon}\left(\mathbb{R}^{n}\right)} \leq C\|f\|_{L^{\frac{n+1}{2}}\left(\mathbb{R}^{n}\right)}
$$

and

$$
\|\mathcal{A} f\|_{L^{\frac{n+1}{n-1}, \frac{n^{2}+n}{n^{2}-n+2}+\epsilon}\left(\mathbb{R}^{n}\right)} \leq C\|f\|_{L^{\frac{n^{2}+n}{n^{2}-n+2}}\left(\mathbb{R}^{n}\right)},
$$

holds for all $f \in C_{c}^{\infty}\left(\mathbb{R}^{n}\right)$ and some $C=C_{p, d, n}$.

The proof of Theorem 1.4 follows the argument of M. Christ in [5], using Theorem 1.1; see [7] for details. 
Notation: Let $A, B$ be complex-valued quantities. We use the notation $A \lesssim B$ or $A=O(B)$ to denote the estimate $|A| \leq C|B|$ where $C$ depends only on $n, d$ and Lebesgue exponents $p$. We use $A \sim B$ to denote the estimates $A \lesssim B \lesssim A$. Finally we will be making various decompositions of an interval into disjoint intervals $\{I\}$ and it will be convenient to keep all intervals $I$ open; therefore, a decomposition $\cup I$ will in fact mean $\cup \bar{I}$.

\section{First stage - the initial Decomposition}

In this section we begin the proof of Theorem 1.1. The main observation is that the algorithm developed in [6] to establish the corresponding inequality for polynomials is robust enough to handle general rational functions with a few modifications. We use the convention that a rational function can be expressed as a ratio of polynomials $R=P / Q$ where $Q$ is not the zero polynomial and we make the basic assumption that $L_{\vec{R}}(t)$ is not the zero rational function; otherwise the proof of Theorem 1.1 is a triviality.

The proof of Theorem 1.1 for polynomials in [6] is carried out in two stages. The extension to rational functions will require three stages. The first stage in [6] is elementary and produces an initial decomposition of any open interval into $O(1)$ open intervals so that on each subinterval a formula relating $J_{\vec{P}}(\vec{t})$ and $L_{\vec{P}}(t)$ holds. This initial decomposition and formula carry over to the setting of rational functions without change. We first require some further notation.

Notation: For any sequence (finite or infinite) of real rational functions $S=$ $\left(S_{1}, S_{2}, \ldots\right)$, we set for any $j \geq 1, \vec{S}_{j}=\left(S_{1}, \ldots, S_{j}\right)$ and

$$
L_{S, j}(t)=L_{S_{1} \ldots S_{j}}(t):=\operatorname{det}\left(\vec{S}_{j}^{\prime}(t) \cdots \vec{S}_{j}^{(j)}(t)\right)
$$

so that in particular, $L_{\vec{R}}=L_{\vec{R}, n}=L_{R_{1} \ldots R_{n}}$ for our original $n$-tuple of rational functions $\vec{R}=\left(R_{1}, \ldots, R_{n}\right)$. For convenience we will often denote $L_{\vec{R}, j}$ simply by $L_{j}$ for $1 \leq j \leq n$.

As in [6] we have the following generalisation of the quotient rule for derivatives.

Lemma 2.1. If $S_{1}, \ldots, S_{k}, R, T$ are $k+2$ real rational functions so that $L_{S_{1} \ldots S_{k} R}$ is not the zero rational function, then

$$
\left(\frac{L_{S_{1} \ldots S_{k} T}}{L_{S_{1} \ldots S_{k} R}}\right)^{\prime}=\frac{L_{S_{1} \ldots S_{k} R T} L_{S_{1} \ldots S_{k}}}{\left[L_{S_{1} \ldots S_{k} R}\right]^{2}} .
$$

And as a consequence we have

Lemma 2.2. If $L_{\vec{R}}=L_{R_{1} \ldots R_{n}}$ is not the zero rational function, then for every $1 \leq j \leq n, L_{\vec{R}, j}=L_{R_{1} \ldots R_{j}}$ is not the zero rational function.

The real roots and real poles of all the rational functions $\left\{L_{\vec{R}, j}\right\}_{j=1}^{n}$ give us our initial decomposition of an arbitrary open interval $I=\cup J$ into $0(1)$ disjoint open intervals so that on each $J$, every $L_{\vec{R}, j}$ is either strictly positive or strictly negative. 
We now write down a formula relating the determinant of the Jacobian matrix for the mapping $\Phi_{\vec{R}}(\vec{t})=\vec{R}\left(t_{1}\right)+\cdots+\vec{R}\left(t_{n}\right), J_{\vec{R}}(\vec{t})=\operatorname{det}\left(\vec{R}^{\prime}\left(t_{1}\right) \cdots \vec{R}^{\prime}\left(t_{n}\right)\right)$, and the rational functions $L_{j}=L_{\vec{R}, j}, 1 \leq j \leq n$ which will be valid on each interval $J$. We will write $J_{\vec{R}}$ as a series of nested iterated integrals. To this end we define a sequence of multi-variate functions $\left\{I_{r}\right\}_{r=1}^{n}$; for each $1 \leq r \leq n, I_{r}=I_{r}\left(x_{1}, \ldots, x_{r}\right)$ will be a function of $r$ variables which will be well defined on $J^{r}$ for each interval $J$ arising in the initial decomposition. We define this sequence inductively. For $r=1$ we set $I_{1}(x)=L_{n-2}(x) L_{n}(x) /\left[L_{n-1}(x)\right]^{2}$ and then inductively, define

$$
\begin{gathered}
I_{r}\left(x_{1}, \ldots, x_{r}\right)= \\
\prod_{s=1}^{r} \frac{L_{n-r-1}\left(x_{s}\right) L_{n-r+1}\left(x_{s}\right)}{\left[L_{n-r}\left(x_{s}\right)\right]^{2}} \int_{x_{1}}^{x_{2}} \ldots \int_{x_{r-1}}^{x_{r}} I_{r-1}\left(y_{1}, \ldots, y_{r-1}\right) d y_{1} . . d y_{r-1} .
\end{gathered}
$$

In order to make sense of $I_{n-1}$ and $I_{n}$ we set $L_{0}=L_{-1} \equiv 1$.

Following the differential calculus argument in [6] verbatim we see that the formula

$$
J_{\vec{R}}\left(t_{1}, \ldots, t_{n}\right)=I_{n}\left(t_{1}, \ldots, t_{n}\right)
$$

holds on each $J^{n}$. When $n=2$ (4) simply states

$$
J_{\vec{R}}(s, t)=L_{1}(s) L_{1}(t) \int_{s}^{t} \frac{L_{2}(w)}{L_{1}^{2}(w)} d w
$$

for any $s, t \in J$ where $J$ is an interval from our initial decomposition. When $n=3$

(4) becomes

$$
J_{\vec{R}}(s, t, u)=L_{1}(s) L_{1}(t) L_{1}(u) \int_{s}^{t} \int_{t}^{u} \frac{L_{2}(v) L_{2}(w)}{\left[L_{1}(v) L_{1}(w)\right]^{2}} \int_{v}^{w} \frac{L_{3}(z) L_{1}(z)}{L_{2}(z)^{2}} d z d w d v
$$

for any $s, t, u \in J$.

\section{SECOnd Stage - Two DeComposition PRocedures}

Our goal now is to use two decomposition procedures to decompose each interval $J$ arising from the initial decomposition into $O(1)$ intervals so that on each subinterval every rational function $L_{\vec{R}, j}, 1 \leq j \leq n$ appearing in the formula (4) for $J_{\vec{R}}(\vec{t})$ behaves like a centred monomial, $L_{\vec{R}, j}(t) \sim A_{j}(t-b)^{k_{j}}$ for some $k_{j} \in \mathbb{Z}$, all with the same centre $b \in \mathbb{R}$ ! In fact we have the following

Proposition 3.1. Let $P_{1}, \ldots, P_{M}$ be $M$ real polynomials with degrees at most $D$. Then any open interval $J=\cup L$ can be decomposed into $O(1)$ (here the implicit constant depends only on $M$ and $D$ ) open intervals so that for every $L$, we have $M$ exponents $k_{1}=k_{1}(M), \ldots, k_{M}=k_{M}(L) \in \mathbb{Z}, M$ nonzero constants $A_{1}=$ $A_{1}(L), \ldots, A_{M}=A_{M}(L)$ and a single centre $b=b(L) \in \mathbb{R}$ where for each $1 \leq j \leq$ $M$, we have $P_{j}(t) \sim A_{j}(t-b)^{k_{j}}$ holding on $L$.

The proof of Proposition 3.1 relies on two decomposition procedures. The first procedure was introduced in [6] and decomposes any open interval $J$ with respect to a given polynomial $Q$. This procedure has the advantage of describing $Q$ over the entire interval $J$. 
$D 1$ Given a real polynomial $Q$. Then $J=\cup I$ can be decomposed into 0(1) open disjoint intervals so that on each

I: $Q(t) \sim A(t-b)^{k}$ for some $A=A_{I} \neq 0$, an integer $k=k_{I} \geq 0$ and $b=b_{I}$, the real part of a root of $Q$.

The second decomposition procedure was introduced in [3] and gives a decomposition which depends not only on a given polynomial $Q$ but also depends on a given centre $b$. Here we will attempt to describe $Q$ on most of $J$ as monomials with varying exponents but with a fixed centre $b$.

D2 Given a real polynomial $Q$ and a centre $b \in \mathbb{R}$. Then $J=\cup I$ can be decomposed into 0(1) open disjoint intervals which fall into two classes: $\mathcal{G}$ (gaps) and $\mathcal{D}$ (dyadic).

On $I \in \mathcal{G}: \quad Q(t) \sim A(t-b)^{k}$ for some $A=A_{I} \neq 0$ and an integer $k=k_{I} \geq 0$.

On $I \in \mathcal{D}: \quad(t-b) \sim B$ for some $B=B_{I} \neq 0$.

Furthermore, if $Q(t+b)=\sum c_{k} t^{k}$ and $c_{k_{0}}=0$ then no gaps $I \in \mathcal{G}$ exist on which $Q(t) \sim A(t-b)^{k_{0}}$.

Using $D 1$ and $D 2$ in tandem we will arrive at a proof of Proposition 3.1 (we will not use the last statement in $D 2$ here but this will be important for the third stage). This will be carried out in $M$ steps. Step 1 is very simple; we apply $D 1$ to $P_{1}$ to obtain a decomposition $J=\cup K$ so that on each

$K: \quad P_{1}(t) \sim A_{1}(t-b)^{k_{1}} \quad$ for some $A_{1}=A_{1}(K) \neq 0, k_{1}=k_{1}(K) \in \mathbb{Z}$ and $b=b(K) \in \mathbb{R}$.

In step 2 we decompose each $K$ further to pin down the behaviour of $P_{2}$. In fact to each $K$, we apply $D 2$ with respect to $P_{2}$ and $b=b(K)$ to obtain a further decomposition $K=\left(\cup L^{\prime}\right) \cup\left(\cup L^{\prime \prime}\right)$ into gaps $L^{\prime}$ and dyadic intervals $L^{\prime \prime}$. On each

gap $L^{\prime}: \quad P_{2}(t) \sim A_{2}(t-b)^{k_{2}}$ for some $A_{2}=A_{2}\left(L^{\prime}\right) \neq 0$ and $k_{2}=k_{2}\left(L^{\prime}\right) \in \mathbb{Z}$ (but $b=b(K)$ does not change).

However on each dyadic $L^{\prime \prime}, t-b \sim B$ for some nonzero $B=B\left(L^{\prime \prime}\right)$ and therefore $P_{1}(t) \sim A_{1} B^{k_{1}}$ on $L^{\prime \prime}$. To complete step 2 , we decompose each dyadic $L^{\prime \prime}=\cup L^{\prime \prime \prime}$ further using $D 1$ with respect to $P_{2}$ so that on each

$L^{\prime \prime \prime}: \quad P_{2}(t) \sim A_{2}(t-c)^{k_{2}}$ for some nonzero $A_{2}=A_{2}\left(L^{\prime \prime \prime}\right), k_{2}=k_{2}\left(L^{\prime \prime \prime}\right) \in \mathbb{Z}$ and $c=c\left(L^{\prime \prime \prime}\right) \in \mathbb{R}$.

The important observation here is that we also have $P_{1}(t) \sim \tilde{A}_{1}(t-c)^{0}$ on $L^{\prime \prime \prime}$ where $\tilde{A}_{1}=A_{1} B^{k_{1}}$ and $c=c\left(L^{\prime \prime \prime}\right)$ as above. This completes step 2. To recapitulate, step 2 has produced a decomposition $K=\cup L$ into $O(1)$ open intervals so that on each 
$L: \quad P_{1}(t) \sim A_{1}(t-b)^{k_{1}}$ and $P_{2}(t) \sim A_{2}(t-b)^{k_{2}}$ for some nonzero constants $A_{1}=A_{1}(L), A_{2}=A_{2}(L)$, exponents $k_{1}=k_{1}(L), k_{2}=k_{2}(L) \in \mathbb{Z}$ and centre $b=b(L) \in \mathbb{R}$.

Step 3 simply repeats step 2 for $P_{3}$, etc... and by the time step $M$ is carried out the proof of Proposition 3.1 finishes.

Of course we could at this stage apply Proposition 3.1 to the sequence of polynomials formed from the numerators and denominators of the rational functions $L_{\vec{R}, j}, 1 \leq$ $j \leq n$ appearing in the series of nested iterated integrals $I_{n}$, making the various integrands of $I_{n}$ comprise of centred monomials all of which have the same centre. However in this way we are treating the rational functions $L_{\vec{R}, j}$ independently (which of course they are not) and consequently we will have no control over the exponents which arise. Without avoiding certain 'bad' exponents the resulting inequality is in fact false. We will see in the third stage how to use the affine invariance of the inequality to guarantee that these bad exponents do not arise. But in order to achieve this (learning various lessons from [12]) it will be important to first stablise the behaviour of the denominators of the original $n$-tuple of rational functions.

This is the second stage which produces a secondary decomposition, decomposing each interval $J$ from the initial decomposition further. Applying Proposition 3.1 to the polynomials arising as denominators $Q_{1}, \ldots, Q_{n}$ from our original $n$-tuple of rational functions $\vec{R}=\left(R_{1}, \ldots, R_{n}\right)$ where $R_{j}=P_{j} / Q_{j}$, we see that every interval $J=\cup K$ from the original decomposition can be decomposed into $O(1)$ disjoint intervals so that on each

$K: \quad Q_{j}(t) \sim A_{j}(t-b)^{\ell_{j}}$ for some nonzero $A_{j}=A_{j}(K), \ell_{j}=\ell_{j}(K) \in \mathbb{Z}$ and $b=b(K) \in \mathbb{R}$.

Thus on $K, \quad\left[Q_{1} \cdots Q_{n}\right](t) \sim B(t-b)^{l_{n}}$ for some nonzero $B=B(K)$ and $l_{n}=$ $l_{n}(K)=\ell_{1}+\cdots+\ell_{n}$. We will use the affine invariance of (1) for $\vec{R}$ to change the rational functions $L_{\vec{R}, j}, 1 \leq j \leq n$ in order to avoid certain bad exponents and we will see that various powers of $Q_{1} \cdots Q_{n}$ will appear as denominators of the transformed $L_{\vec{R}, j}$. For instance we will begin the next section by stablising the behaviour of $L_{\vec{R}, n}$ and we note $N_{\vec{R}, n}:=\left[Q_{1} \cdots Q_{n}\right]^{2^{n}} L_{\vec{R}, j}$ is a polynomial and serves as a numerator for $L_{\vec{R}, n}$.

\section{THIRD STAGE - THE ALGORITHM}

We now describe the general algorithm developed in [6] with the appropriate modifications needed to pass from polynomials to rational functions. The algorithm is carried out in $n$ steps and roughly follows the $M$ steps in the proof of Proposition 3.1 with respect to certain polynomial numerators $N_{\vec{R}, j}$ of $L_{\vec{R}, j}$ (with the order given by $\left.N_{\vec{R}, n}, N_{\vec{R}, 1}, N_{\vec{R}, 2}, \ldots, N_{\vec{R}, n-1}\right)$. However we will keep modifying the $n$ tuple of rational functions $\vec{R}$ by an affine map $\vec{R} \rightarrow A \vec{R}$ at the end of each step (to 
avoid certain bad exponents) before proceeding to the next step. The key which makes this work lies in the feature (which we have not used yet) of $D 2$ that if $Q(t+b)=\sum c_{k} t^{k}$ and $c_{k_{0}}=0$ then no gaps $I \in \mathcal{G}$ exist on which $Q(t) \sim A(t-b)^{k_{0}}$.

As in [6] we will systematically suppress all multiplicative nonzero constants arising in inequalities for polynomials on various intervals; for example we will write $Q(t) \sim$ $(t-b)^{k_{0}}$ instead of $Q(t) \sim A(t-b)^{k_{0}}$ as above. The reader can easily check that these constants always cancel out by homogeneity of the inequality (1) we are trying to establish for rational functions.

Much of this section closely follows [6] but we have decided not to truncate the presentation for the convenience of the reader. Our aim is to decompose an interval $K$ from the secondary decomposition into 0(1) intervals so that on each subinterval (1) holds for $\vec{R}$. Recall that associated to $K$ is a centre $b=b(K)$ and exponent $l_{n}=l_{n}(K)=2^{n}\left[\ell_{1}+\cdots+\ell_{n}\right]$ so that on $K,\left[Q_{1} \cdots Q_{n}\right]^{2^{n}}(t) \sim(t-b)^{l_{n}}$ (we are applying our convention to suppress multiplicative constants).

Step 1: Here we will decompose $K$ into $O(1)$ disjoint open intervals of two types, $T_{0}$ and $T_{1}$. Use $D 2$ with respect to $N_{\vec{R}, n}$ and $b$ to decompose $K=\cup L$ into $0(1)$ gap $(\mathcal{G})$ intervals or dyadic $(\mathcal{D})$ intervals so that on each

$L \in \mathcal{G}: \quad N_{\vec{R}, n}(t) \sim\left(t-b_{0}\right)^{j_{0}}$ for some exponent $j_{0}=j_{0}(L)$ and where $b_{0}=b$.

The intervals $L \in \mathcal{G}$ are the ones of type $T_{0}$. To obtain the intervals of type $T_{1}$ we first observe that on $L \in \mathcal{D}, t-b \sim 1$, and so every denominator $Q_{j}$ is about constant; that is, $Q_{j}(t) \sim 1$ on $L$. Next we apply $D 1$ to $N_{\vec{R}, n}$ to decompose each $L \in \mathcal{D}, L=\cup L^{\prime}$ into $O(1)$ disjoint intervals so that on each

$L^{\prime}: \quad N_{\vec{R}, n}(t) \sim\left(t-b_{1}\right)^{j_{1}}$ for some exponent $j_{1}=j_{1}\left(L^{\prime}\right)$ and some centre $b_{1}=b_{1}\left(L^{\prime}\right)$.

The intervals $L^{\prime}$ are the ones of type $T_{1}$. To recapitulate, in this initial step we have decomposed $K=\cup L$ into $O(1)$ intervals of two types, $T_{0}$ and $T_{1}$. To each interval $L \in T_{r}, r=0,1$ we have associated a centre $b_{r}=b_{r}(L)\left(b_{0}=b\right.$ and $\left.b_{1}=b_{1}(L)\right)$ and an exponent $k_{r}=k_{r}(L)\left(k_{0}=j_{0}(L)-l_{n}(K)\right.$ and $\left.k_{1}=j_{1}(L)\right)$ so that on

$L \in T_{r}: L_{\vec{R}, n}(t) \sim\left(t-b_{r}\right)^{k_{r}}$.

We choose to emphasis the dependence of various quantities on the type, indexed by $r=0,1$, over the dependence on the interval $L \in T_{r}$. Before we proceed to the step 2 we transform $\vec{R}$ in order to avoid certain bad exponents when we apply $D 2$ to a certain $N_{\vec{R}, 1}$ and centre $b_{r}$. To this end we introduce for each $L \in T_{r}, r=0,1$,

$$
A_{r}=A_{r}(L)=\left(\begin{array}{ccc}
a_{1} & \cdots & a_{n} \\
& * &
\end{array}\right) \in G L_{n}(\mathbb{R})
$$

where the row vector $\vec{a}_{r}=\vec{a}_{r}(L)=\left(a_{1}, \ldots, a_{n}\right) \in \mathbb{R}^{n}$ will be chosen momentarily to be nonzero and the remaining entries chosen to guarantee that $A_{r}$ is invertible but otherwise chosen in an arbitrary fashion. We note that $L_{A_{r} \vec{R}, n}=\left(\operatorname{det} A_{r}\right) L_{\vec{R}, n}$ is 
only changed by a multiplicative constant and so will not affect (1). On the other hand,

$$
L_{A_{r} \vec{R}, 1}=\sum_{m=1}^{n} a_{m}\left(P_{m} / Q_{m}\right)^{\prime}=\left(\sum_{m=1}^{n} a_{m} S_{m}\right) /\left[Q_{1} \cdots Q_{n}\right]^{2}
$$

for certain polynomials $S_{m}$. We define $N_{A_{r} \vec{R}, 1}:=\left[Q_{1} \cdots Q_{n}\right]^{2} L_{A_{r} \vec{R}, 1}=\sum_{m=1}^{n} a_{m} S_{m}$.

Thus if $S_{m}\left(t+b_{r}\right)=\sum c_{j}^{m} t^{j}$, then

$$
N_{A_{r} \vec{R}, 1}\left(t+b_{r}\right)=\sum_{j} \vec{a}_{r} \cdot \overrightarrow{c_{j}} t^{j}
$$

where $\overrightarrow{c_{j}}=\left(c_{j}^{1}, \ldots, c_{j}^{n}\right)$. For $L \in T_{0}$, we choose $\vec{a}_{0}=\vec{a}_{0}(L)=\left(a_{1}, \ldots, a_{n}\right)$ to be any nonzero vector which is orthogonal to the hyperplane $H_{0}=\operatorname{span}\left\{\vec{c}_{\mathcal{N}_{0}+m}\right\}_{m=1}^{n-1}$ where $\mathcal{N}_{0}=\mathcal{N}_{0}(L)=\left\lfloor\frac{k_{0}}{n}\right\rfloor+2\left(\ell_{1}+\cdots \ell_{n}\right)$. For $L \in T_{1}$, we choose $\vec{a}_{1}=\vec{a}_{1}(L)=$ $\left(a_{1}, \ldots, a_{n}\right)$ to be any nonzero vector which is orthogonal to the hyperplane $H_{1}=$ $\operatorname{span}\left\{\vec{c}_{\mathcal{N}_{1}+m}\right\}_{m=1}^{n-1}$ where $\mathcal{N}_{1}=\mathcal{N}_{1}(L)=\left\lfloor\frac{k_{1}}{n}\right\rfloor$

Step 2: In this step we will decompose each $L \in T_{r}, r=0,1$ arising from Step 1 and repeat the process with $N_{\vec{R}, n}$ replaced by $N_{A_{r}, \vec{R}, 1}$; thus by the end of this step we will arrive at intervals of four types, $T_{00}, T_{01}, T_{10}$ and $T_{11}$. Fix the type $r \in\{0,1\}$ and interval $L \in T_{r}$ from Step 1 and apply $D 2$ with respect to $N_{A_{r} \vec{R}, 1}$ and $b_{r}$ to decompose $L=\cup M$ into $O(1)$ gap $(\mathcal{G})$ intervals and dyadic $(\mathcal{D})$ intervals. The intervals $M \in \mathcal{G}$ are the intervals of type $T_{r 0}$ and on such an

$M: \quad N_{A_{r} \overrightarrow{R, 1}}(t) \sim\left(t-b_{r 0}\right)^{j_{r 0}}$ for some centre $b_{r 0}$ and exponent $j_{r 0}$. Here the centre $b_{r 0}=b_{r}$ has not changed and most importantly, by our choice of $A_{r}$, the exponent $j_{r 0} \notin\left\{\mathcal{N}_{r}+m\right\}_{m=1}^{n-1}$.

Furthermore on a dyadic interval $M \in \mathcal{D}, \quad t-b_{r} \sim 1$ and so $L_{A_{r} \vec{R}, n}(t) \sim 1$ and well as each denominator $Q_{j}(t) \sim 1$ on $M$. To arrive at the intervals of type $T_{r 1}$, we use $D 1$ with respect to $N_{A_{r} \vec{R}, 1}$ to decompose each dyadic $M=\cup M^{\prime}$ into $O(1)$ disjoint intervals so that on each

$M^{\prime}: \quad N_{A_{r} \vec{R}, 1}(t) \sim\left(t-b_{r 1}\right)^{j_{r 1}}$ for some centre $b_{r 1}=b_{r 1}\left(M^{\prime}\right)$ and exponent $j_{r 1}=$ $j_{r 1}\left(M^{\prime}\right)$. Here we have no control over which exponent $j_{r 1}$ arises. The intervals $M^{\prime}$ are the intervals of type $T_{r 1}$.

To recapitulate, we have decomposed an interval $L$ from Step 1 of type $T_{r}, L=$ $\cup M$ into $O(1)$ intervals of two further types, $T_{r 0}$ and $T_{r 1}$. To each interval $M \in$ $T_{r s}, s=0,1$ we have associated a centre $b_{r s}=b_{r s}(M)\left(b_{r 0}=b_{r}\right)$ and an exponent $k_{r s}=k_{r s}(L)\left(k_{00}=j_{00}-2 l_{n}, k_{10}=j_{10}, k_{01}=j_{01}\right.$ and $\left.k_{11}=j_{11}\right)$ so that on

$M \in T_{r 0}: \quad L_{A_{r} \vec{R}, n}(t) \sim\left(t-b_{r 0}\right)^{k_{r}}, L_{A_{r} \vec{R}, 1}(t) \sim\left(t-b_{r 0}\right)^{k_{r 0}}$ where $b_{r 0}=b_{r}$ and $k_{r 0} \notin\left\{\left\lfloor\frac{k_{r}}{n}\right\rfloor+m\right\}_{m=1}^{n-1}$; and on

$M \in T_{r 1}: L_{A_{r} \vec{R}, n}(t) \sim 1, L_{A_{r} \vec{R}, 1}(t) \sim\left(t-b_{r 1}\right)^{k_{r 1}}$. 
Before we proceed to the next step we transform $A_{r} \vec{R}$ in order to avoid again certain bad exponents when we apply $D 2$ with respect to a certain $N_{A_{r} \vec{R}, 2}$ and $b_{r 0}$. To this end we introduce for each $M \in T_{r s}, r, s \in\{0,1\}$,

$$
A_{r s}=A_{r s}(M)=\left(\begin{array}{c|c}
1 & 0 \\
\hline & a_{1} a_{2} \cdots a_{n-1} \\
0 & *
\end{array}\right) A_{r}
$$

for an appropriate choice of a nonzero $\vec{a}_{r s}=\vec{a}_{r s}(M)=\left(a_{1}, \ldots, a_{n-1}\right)$ which we will momentarily describe. First we note from the form of $A_{r s}, L_{A_{r s} \vec{R}, 1}=L_{A_{r} \vec{R}, 1}$ remains unchanged and $L_{A_{r s} \vec{R}, n}$ changes only by a multiplicative constant.

If $A_{r} \vec{R}=\left(U_{1}, \ldots, U_{n}\right)$ where each $\left[Q_{1} \cdots Q_{n}\right] U_{j}$ is a polynomial, then

$$
L_{A_{r s} \vec{R}, 2}=a_{1} L_{U_{1} U_{2}}+a_{2} L_{U_{1} U_{3}}+\cdots+a_{n-1} L_{U_{1} U_{n}}
$$

and so $L_{A_{r s} \vec{R}, 2}=\sum_{m=1}^{n-1} a_{m} S_{m} /\left[Q_{1} \cdots Q_{n}\right]^{6}$ for certain polynomials $S_{m}$. We define $N_{A_{r s} \vec{R}, 2}:=\left[Q_{1} \cdots Q_{n}\right]^{6} L_{A_{r s} \vec{R}, 2}$. Thus if $S_{m}\left(t+b_{r s}\right)=\sum c_{j}^{m} t^{j}$, then

$$
N_{A_{r s} \vec{R}, 2}\left(t+b_{r s}\right)=\sum_{j} \vec{a}_{r} \cdot \overrightarrow{c_{j}} t^{j}
$$

where $\overrightarrow{c_{j}}=\left(c_{j}^{1}, \ldots, c_{j}^{n-1}\right)$. For $M \in T_{r s}$, we choose $\vec{a}_{r s}=\vec{a}_{r s}(M)=\left(a_{1}, \ldots, a_{n-1}\right)$ to be any nonzero vector which is orthogonal to the subspace spanned by $\left\{\vec{c}_{\mathcal{N}_{r s}+m}\right\}_{m=1}^{n-2}$ where

$\mathcal{N}_{00}=\mathcal{N}_{00}(M)=\left\lfloor\frac{n-2}{n-1} k_{00}+\frac{k_{0}}{n-1}\right\rfloor+6 l_{n}, \quad \mathcal{N}_{10}=\mathcal{N}_{10}(M)=\left\lfloor\frac{n-2}{n-1} k_{10}+\frac{k_{1}}{n-1}\right\rfloor$

and $\mathcal{N}_{r 1}=\mathcal{N}_{r 1}(M)=\left\lfloor\frac{n-2}{n-1} k_{r 1}\right\rfloor$.

Step $m \rightarrow$ Step $(m+1)$ : We now describe how we pass from Step $m$ to Step $(m+1), 2 \leq m \leq n-1$.

The intervals which arise by Step $m$ will be of $2^{m-1}$ types $T_{\mathbf{r}}$, parametrised by 0-1 bitstrings $\mathbf{r}=r_{1} \cdots r_{m-1}$ of length $m-1$. Fix an interval $J$ of type $T_{\mathbf{r}}$; we will have associated to $J$ a centre (real number) $b_{\mathbf{r}}=b_{\mathbf{r}}(J)$ and an exponent (integer) $k_{\mathbf{r}}=k_{\mathbf{r}}(J)$. Furthermore $J$ will have a unique parent (and grandparent, etc... all the way back to an interval from the secondary decomposition) $\tilde{J}$ from the previous step - Step $(m-1)$ - of type $T_{\tilde{\mathbf{r}}}$ where $\tilde{\mathbf{r}}=r_{1} \cdots r_{m-2}$ and there will have been associated to $\tilde{J}$ a matrix $A_{\tilde{\mathbf{r}}}=A_{\tilde{\mathbf{r}}}(\tilde{J}) \in G L_{n}(\mathbb{R})$ so that on

$$
J: \quad L_{A_{\tilde{\mathbf{r}}} \vec{R}, m-2}(t) \sim\left(t-b_{\mathbf{r}}\right)^{k_{\mathbf{r}}} .
$$

When $m=2, \tilde{\mathbf{r}}$ is the empty string and the left hand side of (6) is interpreted as $L_{\vec{R}, n}$. To carry out the decomposition of each interval $J=\cup K$ into intervals of type $T_{\mathbf{r} 0}$ or type $T_{\mathbf{r} 1}$ for Step $(m+1)$, we will need to construct an appropriate invertible matrix $A_{\mathbf{r}}=A_{\mathbf{r}}(J)$ (which in fact will leave the first $m-2$ components of $A_{\tilde{\mathbf{r}}} \vec{R}$ unchanged). For an interval $K$ of type $T_{\mathbf{r} 0}$ we will find a centre $b_{\mathbf{r} 0}=b_{\mathbf{r} 0}(K)$ and an exponent $k_{\mathrm{r} 0}=k_{\mathrm{r} 0}(K)$ so that on

$$
K: \quad L_{A_{\mathbf{r}} \vec{R}, m-1}(t) \sim\left(t-b_{\mathbf{r} 0}\right)^{k_{\mathrm{ro}}} .
$$


Importantly we will achieve this with $b_{\mathbf{r} 0}=b_{\mathbf{r}}$ and some $k_{\mathbf{r} 0} \notin\left\{N_{\mathbf{r}}+1, \ldots, N_{\mathbf{r}}+\right.$ $n-m+1\}$ where, for $m \geq 3, N_{\mathbf{r}}=\left\lfloor\frac{n-m+1}{n-m+2} k_{\mathbf{r}}\right\rfloor$ if $\mathbf{r} \neq r_{1} 0 \cdots 0$ and

$$
N_{\mathbf{r}}=\left\lfloor\frac{n-m+1}{n-m+2} k_{\mathbf{r}}+\frac{k_{r_{1}}}{n-m+2}\right\rfloor \text { if } \mathbf{r}=r_{1} 0 \cdots 0 .
$$

When $m=2, \mathbf{r}=r_{1}$ and $N_{\mathbf{r}}=\left\lfloor\frac{k_{r_{1}}}{n-m+2}\right\rfloor=\left\lfloor\frac{k_{r_{1}}}{n}\right\rfloor$. For an interval $K$ of type $T_{\mathbf{r} 1}$ we will find a centre $b_{\mathbf{r} 1}=b_{\mathbf{r} 1}(K)$ and an exponent $k_{\mathbf{r} 1}=k_{\mathbf{r} 1}(K)$ so that on

$$
K: \quad L_{A_{\mathbf{r}} \Gamma, m-1}(t) \sim\left(t-b_{\mathbf{r} 1}\right)^{k_{\mathrm{r} 1}} .
$$

Here we will have no control over the values of $b_{\mathbf{r} 1}$ and $k_{\mathbf{r} 1}$.

Before we see (7) and (9) we construct the invertible matrix $A_{\mathbf{r}}=A_{\mathbf{r}}(J)$ which will depend on $b_{\mathbf{r}}=b_{\mathbf{r}}(J)$ and $k_{\mathbf{r}}=k_{\mathbf{r}}(J)$ already determined by Step $m$. In fact

$$
A_{\mathbf{r}}=\left(\begin{array}{c|c}
\mathrm{I}_{m-2} & \mathrm{O} \\
\hline \mathrm{O} & a_{1} a_{2} \cdots a_{n-m+2} \\
*
\end{array}\right) A_{\tilde{\mathbf{r}}}
$$

for an appropriate choice of $\vec{a}_{\mathbf{r}}=\vec{a}_{\mathbf{r}}(J)=\left(a_{1}, \ldots, a_{n-m+2}\right) \in \mathbb{R}^{n-m+2}$ which we will momentarily describe. First note that from the form of $A_{\mathbf{r}}, L_{A_{\mathbf{r}} \vec{R}, j}=$ $L_{A_{\tilde{\mathbf{r}}} \vec{R}, j}, 1 \leq j \leq m-2$ (if $m \geq 3$ ) remain unchanged while $L_{A_{\mathbf{r}} \vec{R}, n}$ changes only be a multiplicative constant.

If $A_{\tilde{\mathbf{r}}} \vec{R}=\left(U_{1}, \ldots, U_{n}\right)$ where each $\left[Q_{1} \cdots Q_{n}\right] U_{j}$ is a polynomial, then

$$
L_{A_{\mathbf{r}} \vec{R}, m-1}=\sum_{q=1}^{n-m+2} a_{q} L_{U_{1} \cdots U_{m-2} U_{m-2+q}}
$$

and so $L_{A_{\mathbf{r}} \vec{R}, m-1}=\sum_{q=1}^{n-m+2} a_{q} S_{q} /\left[Q_{1} \cdots Q_{n}\right]^{2^{m}-2}$ for certain polynomials $S_{q}$. We define $N_{A_{\mathbf{r}} \vec{R}, m-1}:=\left[Q_{1} \cdots Q_{n}\right]^{2^{m}-2} L_{A_{\mathbf{r}} \vec{R}, m-1}$. Thus if $S_{q}\left(t+b_{\mathbf{r}}\right)=\sum c_{j}^{q} t^{j}$, then

$$
N_{A_{\mathbf{r}} \vec{R}, m-1}\left(t+b_{\mathbf{r}}\right)=\sum_{j} \vec{a}_{\mathbf{r}} \cdot \overrightarrow{c_{j}} t^{j}
$$

where $\overrightarrow{c_{j}}=\left(c_{j}^{1}, \ldots, c_{j}^{n-m+2}\right)$. For $J \in T_{\mathbf{r}}$, we choose $\vec{a}_{\mathbf{r}}=\vec{a}_{\mathbf{r}}(J)=\left(a_{1}, \ldots, a_{n-m+2}\right)$ to be any nonzero vector which is orthogonal to the subspace spanned by $\left\{\vec{c}_{\mathcal{N}_{\mathbf{r}}+m}\right\}_{m=1}^{n-m+1}$ where $\mathcal{N}_{\mathbf{r}}=N_{\mathbf{r}}$ if $\mathbf{r} \neq 0 \cdots 0$ and $\mathcal{N}_{\mathbf{r}}=N_{\mathbf{r}}+\left[2^{m}-2\right] l_{n}$ if $\mathbf{r}=0 \cdots 0\left(N_{\mathbf{r}}\right.$ as in (8).

The procedure to establish (7) and (9) is exactly the same as in Steps 1 and 2; use $D 2$ with respect to the polynomial $N_{A_{\mathbf{r}} \vec{R}, m-1}$ and centre $b_{\mathbf{r}}$ to decompose $J=\cup K$ into gap $(\mathcal{G})$ intervals or dyadic $(\mathcal{D})$ intervals. Note that by construction (7) is satisfied for our gap intervals $K \in \mathcal{G}$ since $N_{A_{\mathbf{r}} \vec{R}, m-1}\left(t+b_{\mathbf{r}}\right)=\sum c_{j} t^{j}$ has the property that the coefficients $c_{\mathcal{N}_{\mathbf{r}}+\ell}$ vanish for all $\ell=1,2, \ldots n-m+1$. The way we defined $A_{\mathrm{r}}$ guarantees that this is the case. Hence these gap intervals will be our intervals of type $T_{\mathrm{r} 0}$. To arrive at our intervals of type $T_{\mathbf{r} 1}$ we use $D 1$ with respect to the polynomial $N_{A_{\mathbf{r}} \vec{R}, m-1}$ to decompose each dyadic $K=\cup K^{\prime}$ further into 0(1) disjoint intervals so that on each $K^{\prime}(9)$ holds. This completes the inductive step from Step $m$ to Step $(m+1)$. 
Step $n$ : We finally arrive at the final step. Let us fix an interval $J_{\mathbf{r}}, \mathbf{r}=r_{1} \cdots r_{n}$, of type $T_{\mathbf{r}}$ at this final step and describe what the algorithm produces on this interval. To this end let $\mathbf{r}_{j}=r_{1} \cdots r_{j}$ when $1 \leq j \leq n$ (so that $\mathbf{r}_{n}=\mathbf{r}$ ) and let $\mathbf{r}_{0}$ denote the empty string. We have $n-1$ invertible matrices $\left\{A_{\mathbf{r}_{1}}, \ldots, A_{\mathbf{r}_{n-1}}\right\}$, $n$ centres $\left\{b_{\mathbf{r}_{1}}, \ldots, b_{\mathbf{r}_{n}}=b_{\mathbf{r}}\right\}$ and $n$ exponents $\left\{k_{\mathbf{r}_{1}}, \ldots, k_{\mathbf{r}}\right\}$ associated to $J_{\mathbf{r}}$, its parent, grandparent, etc... all the way back to an interval $J$ from the secondary decomposition (note there is no matrix $A_{\mathbf{r}}$ as we do not pass from Step $n$ to Step $(n+1))$. Let $0 \leq m \leq n$ be so that $\mathbf{r}=r_{1} \cdots r_{m} 0 \cdots 0$ and $r_{m}=1(m=0$ being the case $\mathbf{r}=0 \cdots 0$ ). When $m \geq 2$ we have the following properties from our algorithm:

Property 1 On $J_{\mathbf{r}}$,

$$
\begin{aligned}
& L_{\vec{R}, n}(t) \sim 1, \ldots, L_{A_{\mathbf{r}_{m-2}} \overrightarrow{R, m-2}}(t) \sim 1, L_{A_{\mathbf{r}_{m-1}} \overrightarrow{R, m-1}}(t) \sim\left(t-b_{\mathbf{r}_{m}}\right)^{k_{\mathbf{r}_{m}}}, \\
& L_{A_{\mathbf{r}_{m}} \overrightarrow{R, m}}(t) \sim\left(t-b_{\mathbf{r}_{m+1}}\right)^{k_{\mathbf{r}_{m+1}}}, \ldots, L_{A_{\mathbf{r}_{n-1}} \vec{R}, n-1}(t) \sim\left(t-b_{\mathbf{r}}\right)^{k_{\mathbf{r}}}
\end{aligned}
$$

where $k_{\mathbf{r}_{m}} \in \mathbb{Z}$ is unrestricted but for $m+1 \leq j \leq n$,

$$
k_{\mathbf{r}_{j}} \notin\left\{N_{\mathbf{r}_{j-1}}+1, \ldots, N_{\mathbf{r}_{j-1}}+n-j+1\right\} \text { where } N_{\mathbf{r}_{j-1}}=\left\lfloor\frac{n-j+1}{n-j+2} k_{\mathbf{r}_{j-1}}\right\rfloor
$$

(the $m=2$ case being interpreted as $L_{\vec{R}, n} \sim 1, L_{A_{\mathbf{r}_{1}} \vec{R}, 1}(t) \sim\left(t-b_{\mathbf{r}_{1} 1}\right)^{k_{\mathbf{r}_{1} 1}}$, etc...).

Property 2 For each $1 \leq j \leq n-1, L_{A_{\mathbf{r}_{j}} \vec{R}, j}=L_{A_{\mathbf{r}_{n-1}} \vec{R}, j}$ because of the form of the matrices $A_{\mathbf{r}_{j}}$. Hence on $J_{\mathbf{r}}$, if $Q=A_{\mathbf{r}_{n-1}} \vec{R}$

$$
\begin{gathered}
L_{Q, n}(t) \sim 1, L_{Q, 1}(t) \sim 1, \ldots, L_{Q, m-1}(t) \sim 1 \\
L_{Q, m}(t) \sim\left(t-b_{\mathbf{r}_{m+1}}\right)^{k_{\mathbf{r}_{m+1}}}, \ldots, L_{Q, n-1}(t) \sim\left(t-b_{\mathbf{r}}\right)^{k_{\mathbf{r}}} .
\end{gathered}
$$

Property 3 For $m \leq j \leq n, \quad b_{\mathbf{r}_{j}}=b_{\mathbf{r}}$.

The cases $m=0$ and $m=1$ are special; here $\mathbf{r}=r_{1} 0 \cdots 0$. In this case we have on $J_{\mathbf{r}}$ :

$$
L_{Q, n}(t) \sim\left(t-b_{r_{1}}\right)^{k_{r_{1}}}, L_{Q, 1}(t) \sim\left(t-b_{r_{1}}\right)^{k_{\mathbf{r}_{2}}}, \ldots, L_{Q, n-1}(t) \sim\left(t-b_{r_{1}}\right)^{k_{\mathbf{r}}}
$$

where $k_{r_{1}} \in \mathbb{Z}$ is unrestricted but each $k_{\mathbf{r}_{j}}, 2 \leq j \leq n$ has the restriction $k_{\mathbf{r}_{j}} \notin$ $\left\{M_{\mathbf{r}_{j-1}}+1, \ldots, M_{\mathbf{r}_{j-1}}+n-j+1\right\}$ where $M_{\mathbf{r}_{j-1}}=\left\lfloor\frac{n-j+1}{d-j+2} k_{\mathbf{r}_{j-1}}+\frac{k_{r_{1}}}{n-j+2}\right\rfloor$ (here $\left.k_{\mathbf{r}_{1}}=0\right)$.

We are now in a position to describe our final decomposition of $I=\cup J$ of any open interval $I$ into $0(1)$ disjoint open subintervals so that (1) for $\vec{R}$ holds on each $J$. The initial and secondary decompositions together with the algorithm set out in this section produce a decomposition of $I=\cup J$ so that Properties 1,2 and 3 hold on each $J$ (this is the case when $m \geq 2$; property (10) holding for the cases $m=0$ and $m=1)$. Now collect together all the centres $\left\{b_{\mathbf{r}}\right\}$ associated to each $J$, its parent, grandparent, etc... (there are 0(1) such centres) and decompose each $J$ 
into disjoint open intervals avoiding these real numbers. Thus we finally arrive at our desired final decomposition for $I$.

\section{Proof of Theorem 1.1}

We now follow [6] and apply Properties 1,2 and 3 to reduce (1) for $\vec{R}$ to establishing a concrete inequality on each interval $J$ from the final decomposition. We begin with the cases $2 \leq m \leq n$; for $q \geq 2$, we change notation slightly and start with any sequence of $q-1$ integers $k_{0}, k_{1}, \ldots, k_{q-2}$ where $k_{0}$ is unrestricted but for $1 \leq j \leq q-2$,

$$
\text { either } k_{j} \leq \frac{q-j-1}{q-j} k_{j-1} \text { or } k_{j} \geq \frac{q-j-1}{q-j} k_{j-1}+(q-j-1) .
$$

One can easily check that the interval of values of $k_{j}$ which are prohibited above (in terms of $k_{j-1}$ ) are the ones avoided in the algorithm of the previous section, thanks to the affine invariance of (1). We will see momentarily why we chose to avoid these particular values.

From this sequence $k_{j}$ we form a new sequence $\sigma_{0}=k_{1}-2 k_{0}, \sigma_{1}=k_{2}+k_{0}-$ $2 k_{1}, \ldots, \sigma_{q-2}=k_{q-3}-2 k_{q-2}$ of integers. We now write down a nested series of iterated integrals. Set $E_{q}=E_{q}\left(x_{1}, \ldots, x_{q}, b\right)$ where

$$
E_{q}=\prod_{r=1}^{q}\left|x_{r}-b\right|^{k_{0}} \int_{x_{1}}^{x_{2}} \cdots \int_{x_{q-1}}^{x_{q}} \prod_{r=1}^{q-1}\left|y_{r}-b\right|^{\sigma_{0}} \cdots \int_{u_{1}}^{u_{2}}|w-b|^{\sigma_{q-2}} d w d u_{1} \cdots d y_{q-1} .
$$

Our desired inequality in this case is implied by the following proposition (see [6]).

Proposition 5.1. For any $q \geq 2, x_{1}<x_{2}<\cdots x_{q}$ and $b \notin\left[x_{1}, x_{q}\right]$,

$$
E_{q} \gtrsim \prod_{r<s}\left(x_{s}-x_{r}\right)
$$

The cases $m=0$ and $m=1$ reduce to a slight variant of Proposition 5.1. Here we start with a sequence of $n$ integers (slightly changing notation again) $k_{0}, \ldots, k_{n-2}$ and $k$ where now $k$ is unrestricted but for $0 \leq j \leq n-2\left(k_{-1}=0\right)$,

$$
k_{j} \leq \frac{n-j-1}{n-j} k_{j-1}+\frac{k}{n-j} \quad \text { or } \quad k_{j} \geq \frac{n-j-1}{n-j} k_{j-1}+\frac{k}{n-j}+(n-j-1) \text {. }
$$

We define a sequence $\tilde{\sigma}_{j}=\sigma_{j}$ for $0 \leq j \leq n-3$ (where the $\sigma_{j}$ are defined above) but we define $\tilde{\sigma}_{n-2}=k+k_{n-3}-2 k_{n-2}$. Finally we define $F_{n}=F_{n}\left(x_{1}, \ldots, x_{n}, b\right)$ exactly as we defined $E_{q}$ with $q=n$ except the sequence $\left\{\sigma_{j}\right\}$ is replaced by $\left\{\tilde{\sigma}_{j}\right\}$. Our desired inequality in these cases follow from the next proposition (again see $[6])$.

Proposition 5.2. For any $x_{1}<x_{2}<\cdots x_{n}$ and $b \notin\left[x_{1}, x_{n}\right]$,

$$
\left|F_{n}\right| \gtrsim \prod_{r=1}^{n}\left|x_{r}-b\right|^{k / n} \prod_{r<s}\left|x_{s}-x_{r}\right| \text {. }
$$


For the proof of Propositions 5.1 and 5.2 we will need to examine iterated integrals of the form

$$
I=\int_{z_{1}}^{z_{2}} \cdots \int_{z_{\ell-1}}^{z_{\ell}} \prod_{r=1}^{\ell-1}\left|y_{r}-b\right|^{\rho_{r}} \prod_{r<s}\left|y_{r}-y_{s}\right| d y_{1} . . d y_{\ell-1}
$$

where $z_{1}<\cdots<z_{\ell}<b$.

One important case to consider is where all the exponents $\rho_{r}$ are equal (a proof of the following lemma can be found in [6]).

Lemma 5.3. If $\rho_{1}=\rho_{2}=\cdots=\rho_{\ell-1}=\rho$,

$$
I \gtrsim \prod_{r=1}^{\ell}\left|z_{r}-b\right|^{\frac{\ell-1}{\ell} \rho} \prod_{r<s}\left|z_{r}-z_{s}\right|
$$

holds if and only if $\rho \geq 0$ or $\rho \leq-\ell$.

We now return to $E_{q}$ and $F_{n}$ in Propositions 5.1 and 5.2 and prove an estimate for these nested series of iterated integrals by making repeated use of Lemma 5.3. We start with the innermost integral and apply Lemma 5.3 to it;

$$
\int_{u_{1}}^{u_{2}}|w-b|^{s} d w \gtrsim\left|u_{1}-u_{2}\right|\left[\left|u_{1}-b\right|\left|u_{2}-b\right|\right]^{\frac{1}{2} s}
$$

holds if and only if $s \geq 0$ or $s \leq-2$. For $E_{q}, s=\sigma_{q-2}=k_{q-3}-2 k_{q-2}$ and by (11) either

i) $k_{q-2} \leq \frac{1}{2} k_{q-3} \Rightarrow s=\sigma_{q-2} \geq 0 \quad$ or

ii) $k_{q-2} \geq \frac{1}{2} k_{q-3}+1 \Rightarrow s=\sigma_{q-2} \leq-2$.

For $F_{n}, s=\tilde{\sigma}_{n-2}=k+k_{n-3}-2 k_{n-2}$ and by (12) either

i) $k_{n-2} \leq \frac{1}{2} k_{n-3}+\frac{k}{2} \Rightarrow s=\tilde{\sigma}_{n-2} \geq 0$ or

ii) $k_{n-2} \geq \frac{1}{2} k_{n-3}+\frac{k}{2}+1 \Rightarrow s=\tilde{\sigma}_{n-2} \leq-2$.

Observe that when we apply Lemma 5.3 iteratively to each successive nested iterated integral defining either $E_{q}$ or $F_{n}$ we end up with an iterated integral with the form $I$ above where all the exponents $\rho_{r}$ are equal and so Lemma 5.3 can once again be applied. At the $(\ell-1)$ th application $(2 \leq \ell \leq q$ or $n)$ of Lemma 5.3 we need to estimate

$$
I_{\ell}=\int_{z_{1}}^{z_{2}} \cdots \int_{z_{\ell-1}}^{z_{\ell}} \prod_{r<t}\left|y_{r}-y_{t}\right| \prod_{r=1}^{\ell-1}\left|y_{r}-b\right|^{\rho_{\ell}} d y_{1} . . d y_{\ell-1}
$$


where $\rho_{\ell}=s_{q-\ell}+\frac{\ell-2}{\ell-1}\left(s_{q-\ell+1}+\cdots+\frac{2}{3}\left(s_{q-3}+\frac{1}{2} s_{q-2}\right)\right)$ and $s=\sigma$ for $E_{q}$ and $s=\tilde{\sigma}$ for $F_{n}$ (and then $q=n$ ).

Claim 1: For $E_{q}($ and so $s=\sigma), \rho_{\ell}=k_{q-\ell-1}-\frac{\ell}{\ell-1} k_{q-\ell}, \quad 2 \leq \ell \leq q$.

Here we interpret $k_{-1}=0$. To prove this claim we proceed by induction on $\ell$; the case $\ell=2$ being clear. By induction, for $3 \leq \ell \leq q, \rho_{\ell}=$

$\sigma_{q-\ell}+\frac{\ell-2}{\ell-1}\left(k_{q-\ell}-\frac{\ell-1}{\ell-2} k_{q-\ell+1}\right)=k_{q-\ell-1}+k_{q-\ell+1}-2 k_{q-\ell}+\frac{\ell-2}{\ell-1} k_{q-\ell}-k_{q-\ell+1}$ and so $\rho_{\ell}=k_{q-\ell-1}-(\ell / \ell-1) k_{q-\ell}$.

By (11) we see that Claim 1 implies that either $\rho_{\ell} \geq 0$ or $\rho_{\ell} \leq-\ell$ only if $2 \leq \ell \leq q-1$ and so Lemma 5.3 can be applied to these $I_{\ell}$.

Claim 2: For $F_{n}($ and so $s=\tilde{\sigma}), \rho_{\ell}=k_{n-\ell-1}-\frac{1}{\ell-1}\left(\ell k_{n-\ell}-k\right), 2 \leq \ell \leq n$.

The proof of Claim 2 is the same as Claim 1, proceeding by induction on $\ell$. Hence by (12), Claim 2 implies that either $\rho_{\ell} \geq 0$ or $\rho_{\ell} \leq-\ell$ for all $2 \leq \ell \leq n$ and so Lemma 5.3 can be applied to all the iterated integrals defining $F_{n}$ giving us the desired estimate for $F_{n}$, completing the proof of Proposition 5.2.

On the other hand, after the $(q-1)$ st application of Lemma 5.3 to each of the iterated integrals defining $E_{q}$ we have

$$
E_{q} \gtrsim \prod_{r=1}^{q}\left|x_{r}-b\right|^{k_{0}} \int_{x_{1}}^{x_{2}} \cdots \int_{x_{q-1}}^{x_{q}} \prod_{r=1}^{q-1}\left|y_{r}-b\right|^{-\frac{q}{q-1} k_{0}} \prod_{r<s}\left|y_{r}-y_{s}\right| d y_{1} . . d y_{q-1} .
$$

Unfortunately the exponent $k_{0}$ is unrestricted, preventing us to obtain an unconditional estimate for $E_{q}$. Nevertheless if $k_{0} \leq 0$ or $k_{0} \geq q-1$, then Lemma 5.3 can be applied once more to conclude $E_{q} \gtrsim \prod_{r<s}\left|x_{r}-x_{s}\right|$, completing the proof of Proposition 5.1 in this case.

It still remains to prove Proposition 5.1 but, from above, we may assume $1 \leq k_{0} \leq$ $q-2$. It turns out that even with this reduction the proof of Proposition 5.1 involves an intricate combinatorial argument but follows exactly as in [6]; we simply note that that the nonnegativity of the sequence $\left\{k_{j}\right\}$ was not used there, only the fact that it is a sequence of integers (positive or negative) was important and crucial. 


\section{REFERENCES}

[1] J.G. Bak, D. Oberlin, and A. Seeger, Restriction of Fourier transforms to curves and related oscillatory integrals, to appear in the Amer. J. Math.

[2] J.G. Bak, D. Oberlin, and A. Seeger, Restriction of Fourier transforms to curves II: some classes with vanishing torsion, to appear in the Journal of the Australian Mathematical Society.

[3] A. Carbery, F. Ricci, and J. Wright, Maximal functions and Hilbert transforms associated to polynomials, Rev. Mat. Iberoamericana 14 (1998), no. 1, 117-144.

[4] M. Christ, On the restriction of the Fourier transform to curves: endpoint results and degenerate cases, Trans. Amer. Math. Soc. 287 (1985), 223-238.

[5] M. Christ, Convolution, curvature and combinatorics: a case study, Int. Math. Res. Notices 1998, no. 19, 1033-1048.

[6] S. Dendrinos, J. Wright, Fourier restriction to polynomial curves I: A geometric inequality, preprint.

[7] S. Dendrinos, N. Laghi and J. Wright, Universal $L^{p}$ improving for averages along polynomial curves in low dimensions, preprint.

[8] S.W. Drury, Restriction of Fourier transforms to curves, Ann. Inst. Fourier, 35 (1985), 117-123.

[9] S.W. Drury, Degenerate curves and harmonic analysis, Math. Proc. Cambridge Philos. Soc. 108 (1990), 89-96.

[10] S.W. Drury, and B. Marshall, Fourier restriction theorems for curves with affine and Euclidean arclengths, Math. Proc. Cambridge Philos. Soc. 97 (1985), 111-125.

[11] S.W. Drury, and B. Marshall, Fourier restriction theorems for degenerate curves, Math. Proc. Cambridge Philos. Soc. 101 (1987), 541-553.

[12] M. Folch-Gabayet, and J. Wright, Singular integral operators associated to curves with rational components, Trans. Amer. Math. Soc. 360 (2008), 1661-1679.

[13] D. Oberlin, Fourier restriction estimates for affine arclength measures in the plane, Proc. Amer. Math. Soc. 129 (2001), 3303-3305.

[14] D. Oberlin, Convolution with measures on polynomial curves, Math. Scand. 90 (2002), no. $1,126-138$.

[15] P. Sjölin, Fourier multipliers and estimates for the Fourier transform of measures carried by smooth curves in $\mathbb{R}^{2}$, Studia Math. 51 (1974), 169-182.

Department of Mathematics, University of Bristol, University Walk, Bristol BS8 1TW, UNITED KINGDOM

E-mail address: S.Dendrinos@bristol.ac.uk

Instituto de Matemáticas, UNAM, Area de la Investigación Científica, Circuito ExteRior, Ciudad Universitaria, México, D.F. 04510

E-mail address: folchgab@matem.unam.mx

School of Mathematics and the Maxwell Institute for Mathematical Sciences, The University of Edinburgh, JCMB, King's Buildings, Edinburgh EH9 3JZ, United Kingdom

E-mail address: J.R.Wright@ed.ac.uk 